prednisolone useful. Patients who have been controlled by oral corticosteroids for a long time and are then successfully weaned off these on to beclomethasone alone should continue to carry their steroid card and should have a supply of steroid tablets at home in case they develop an acute attack of asthma.

\section{OTHER TREATMENTS}

The $\beta$-adrenergic receptor blocking drugs are absolutely contraindicated in asthma. I do not use corticotrophin, antihistamines, expectorants, antitussives, psychotropic drugs, sedatives, or mixtures of ephedrine, theophylline, and a barbiturate in the management of asthma. I have no experience of acupuncture, biofeedback, breathing exercises, hypnotherapy, or group therapy, and, although some individuals might obtain benefit from these, I would not generally recommend them to patients.

Exercise should be encouraged so far as is practicable, especially in children. Exercise-induced asthma may usually be controlled by prior inhalation of DSCG or a sympathomimetic, and it is preferable to prescribe these agents and allow exercise than to have a child feel isolated and handicapped.

\section{ACUTE SEVERE ATTACKS OF ASTHMA}

It is foolish to apply any definition of "status asthmaticus" which requires symptoms to be present for more than 24 hours; patients with acute symptoms may die within a few hours of the onset of an attack. Patients must be aware that they must seek medical advice if they develop an attack that fails to improve after treatment with their usual drugs. This is especially important if the attack is severe enough to confine them to a chair during the day or to keep them awake at night. In such cases the pulse rate will usually be greater than $120 / \mathrm{min}$ and the PEFR less than $100 \mathrm{l} / \mathrm{min}$. Such an attack is best treated in hospital, but before transferring the patient $250 \mathrm{mg}$ of aminophylline may be injected slowly intravenously, together with a bolus intravenous injection of $300 \mathrm{mg}$ hydrocortisone. In hospital the spirometry and blood gases can be monitored and treatment continued with bronchodilators, corticosteroids, oxygen, and rehydration. Eventually ventilation may be required if there is exhaustion and carbon dioxide retention.

\section{Conclusion}

To manage asthma correctly frequent clinical and physiological assessment may be necessary. Although simple, the peak flow meter can give valuable information when following progress. Treatment should be the minimum necessary to control attacks, but the more effective remedies must not be withheld when the clinical condition deteriorates.

\section{Further reading}

Clark, T J H and Godfrey, S, editors, Asthma. London, Chapman and Hall, 1977.

\title{
How to do it
}

\section{Take an examination paper}

\author{
P R FLEMING
}

British Medical fournal, 1978, 2, 1210-1211

Examinations in medicine today include an increasing variety of written papers. The answers required range from lengthy essays to the candidate's marks on specially prepared answer sheets which indicate his responses to multiple-choice questions. First, I will consider those questions that require from the candidate an attempt to convey his thoughts to the examiners in continuous prose. Later, I will briefly consider questions requiring short answers, and multiple-choice questions.

\section{Essay papers}

The traditional essay paper consists of 4-6 questions, on each of which you are expected to write for 30-45 minutes; recently, papers have sometimes included a much larger number of questions, perhaps 15 or 20 , to which the answers must be

Westminster Medical School, London SW1P 2AP

P R FLEMING, MD, FRCP, senior lecturer correspondingly shorter. Sometimes these two types of question appear in the same paper and, for this reason (and for many others), it is essential that you read through the instructions printed on the paper so that you are quite clear about what is wanted. Having read the whole paper through and, incidently, noted whether any, or all, of the questions are compulsory, you should spend a moment or two planning your campaign. If possible, it is clearly desirable to decide at the outset which of several optional questions you will answer but, if the choice is difficult and the minutes are ticking away, it may well be wise to start writing answers to the questions that you have definitely decided to attempt. While you are writing, and however hard you may be concentrating on one question, you may find that ideas and facts about other questions spring to mind, and, if you make a note of them as they arise, you may be able to decide more easily which of the remaining questions to answer.

Whether or not you have to choose which questions to answer, you must be certain, before you start to write, how much time you can afford to spend on each question. At all costs you must answer the right number of questions, and examiners are rarely impressed by the answer consisting of one or two lines and ending in mid-sentence-a ploy that seems designed to give the impression that, had five minutes more been available, a brilliant vignette of the topic under discussion would not have been lost to posterity. 
Something should be said about the composition of the answers themselves. You should study the wording of the question to determine precisely what it is that the examiners require. Unfortunately, this is not always easy but it can usually be assumed, in a traditional "long essay" question, that "Discuss ..." or "Give an account of ..." really mean, "Write all you know about. ..." Difficulties may arise, however, when only a brief answer is required. I must admit that some examiners are not yet very experienced in setting questions which could, in theory, be answered perfectly in 10 minutes, and for the time being you will sometimes have the difficult task of deciding what you (and, you hope, the examiners) regard as essential in such an answer. Nevertheless, most such questions are carefully worded and if, for example, you are trying to "Give an account of the clinical manifestations of chronic renal failure" do not waste time on a discussion of the biochemical disorder.

Candidates are usually reminded of the importance of writing good English but I must admit that most examiners, conscious perhaps of their own limitations, pay little attention to this and regard split infinitives with some equanimity. This is not to say, however, that they will not be irritated by gross solecisms in style and spelling, and statements like "the patient should be P R'D to exclude malaena" are unlikely to improve your chances. Some examiners are touchy too about abbreviations, and you should be careful about their use. Such terms as BP, JVP, and MSU are probably acceptable but you should avoid "statements" like "After g-i bleed BV $\downarrow \rightarrow$ CVP $\downarrow$ " as a substitute for "After gastrointestinal haemorrhage, the fall in circulating blood volume is reflected in a low central venous pressure."

\section{Short answers and MCQ}

The format of some recently introduced written examinations is such that candidates' answers consist of a few lines, or sometimes a phrase or even a single word. Such examinations include the Modified Essay Questions used by the Royal College of General Practitioners, and the written section fo Part II of the MRCP. There are therefore few problems in composing the answers, but it is particularly important for you to pay attention to any instructions and to the precise wording of the questions. In the membership, for example, the questions are very specific and, if you are asked for two possible explanations of a set of laboratory data, there is no point whatever in listing three. Brilliant candidates, who may feel constrained by the unreality of this format, should remind themselves that they will have ample opportunity to show their expertise in other parts of the examination.

The charge of unreality has also often been levelled at multiple-choice questions (MCQ) and the point is well taken, particularly by those with considerable experience in constructing them. Nevertheless, the disadvantages of MCQ are outweighed by the precision with which your factual knowledge may be assessed by this technique, and MCQ have certainly come to stay. This being the case, you must learn how best to do yourself justice in such an examination. Reading the questions carefully is important; the standard of MCQ has risen considerably in recent years and in well-conducted examinations ambiguous questions are now rare. It is equally important for you to come to terms with the problem of how much to guess in answering MCQ. If, as is commonly the case, marks are deducted for wrong answers, you may be reluctant to attempt any questions other than those about which you are certain. Such timidity usually means doing yourself less than justice. Wild guessing is pointless, but, just as action in clinical medicine may be necessary without complete information, so candidates should be prepared to "play their hunches" when answering MCQ. On the not unreasonable assumption that hunches are, on balance, more often right than wrong, and, provided that no more marks are deducted for an incorrect answer than are awarded for a correct one, this practice will almost always result in an improvement in your score.

Eventually this series will be collected into a book and hence no reprints will be available from the authors.
A man in his 50s with coeliac disease-well-controlled for 19 years with a gluten-free diet-has had increasingly frequent attacks of nocturnal epigastric pain and accompanying borborygmi. Is there any explanation or treatment for these symptoms?

The first possibility is that he is unsuspectingly eating some gluten. For example, a firm may have changed the composition of a proprietary food. A careful dietary check, aided by the helpful information of the Coeliac Society (PO Box 181, London NW2 2QY), should be made. Secondly (and perhaps most likely) he may have an abdominal problem unrelated to his coeliac disease. Patients with coeliac disease may develop peptic ulcers, pancreatitis, or the irritable bowel syndrome like anyone else, and the usual diagnostic process must be followed. Thirdly, coeliac patients are more likely to develop intestinal lymphoma than healthy individuals, though the risk is small. Diagnosis of this may be difficult at an early stage, but careful small-intestinal radiology and a jejunal biopsy should be done. Laparotomy may be needed to establish a certain diagnosis.

I have been told that chewing or eating the leaves of feverfew plant can prevent recurrent attacks of migraine. Is there any pharmacological evidence to support this?

Feverfew (syn featherfew, featherfoil) is a perennial wild flower belonging to the family Compositae. An infusion of the plant (either the whole herb or the flower heads) is used in homoeopathy for migraine, alimentary spasms, nervous sickness, as a febrifuge, and to promote or control menstruation. The active constituent is an essentia oil (a mixture of esters, aldehydes, alcohols, ketones, and terpenes). The oil is said to contain camphor, borneol, various esters, and a bitter substance. It is, however, closely related to the camomiles and is used in homoeopathy for the same purpose as Roman camomile. The essential oil from Roman camomile consists mainly of esters of angelic and tiglic acid, isopropyl and amyl alcohols, a camphoranthemone, and anthemic acid, which is responsible for the bitter taste. The flowers, but not the leaves, also contain inositol. Amyl and isopropyl alcohols are vasodilators and, if present in feverfew, could possibly be the basis of its prophylactic effect in migraine. The pharmacological properties of feverfew in relation to its beneficial effect in migraine are being investigated. ${ }^{1}$

${ }^{1}$ Medical News, 16 March 1977.

$A$ woman of 65 had a cerebrovascular accident with right hemiplegia. Subsequently she sustained a fractured femur, and the radiograph showed some osteoporosis. She drinks a daily pint of milk. Is additional calcium of any value?

A pint of milk contains about $500 \mathrm{mg}$ of calcium, and, with a reasonable intake of green vegetables and bread, probably provides all the calcium needed by a woman of 65 , even though people absorb relatively less calcium as they grow older. The osteoporosis may relate to mild vitamin $\mathrm{D}$ deficiency, particularly in the elderly housebound who get no sunlight. Simple dietary deficiency of calcium does not cause osteoporosis, for intakes as high as $1500 \mathrm{mg}$ a day do not protect against bone loss, nor do intakes as low as $300 \mathrm{mg}$ a day lead to increased bone loss. It would do no harm to offer the patient a daily vitamin pill containing vitamins $A$ and $D$. Various commercial preparations contain 100-500 IU of vitamin D. The cheapest and most satisfactory is probably Abidec. One capsule a day supplies 400 IU. A year's treatment costs less than $£ 3$. Even this is not strictly necessary provided the patient continues to walk and gets a reasonable amount of exposure to sunlight in the open air. 